$\begin{array}{ll}\text { Balkanologie } & \begin{array}{l}\text { Balkanologie } \\ \text { Revue d'études pluridisciplinaires }\end{array}\end{array}$

Vol. XI, $n^{\circ}$ 1-2 | 2008

Volume XI Numéro 1-2

\title{
Mobilités du travail (gurbet), stratégies sociales et familiales : Une étude de cas dans les Balkans centraux
}

\section{Petko Hristov}

Traducteur : Nadège Ragaru

\section{OpenEdition \\ Journals}

\section{Édition électronique}

URL : https://journals.openedition.org/balkanologie/912

DOI : 10.4000/balkanologie.912

ISSN : 1965-0582

\section{Éditeur}

Association française d'études sur les Balkans (Afebalk)

\section{Référence électronique}

Petko Hristov, « Mobilités du travail (gurbet), stratégies sociales et familiales : Une étude de cas dans les Balkans centraux », Balkanologie [En ligne], Vol. XI, n 1-2 | 2008, mis en ligne le 31 décembre 2008 consulté le 28 juin 2022. URL : http://journals.openedition.org/balkanologie/912 ; DOI : https://doi.org/ 10.4000/balkanologie.912

Ce document a été généré automatiquement le 17 décembre 2020.

(c) Tous droits réservés 


\title{
Mobilités du travail (gurbet), stratégies sociales et familiales : Une étude de cas dans les Balkans centraux
}

\author{
Petko Hristov \\ Traduction : Nadège Ragaru
}

L'étude des caractéristiques socioculturelles de la vie familiale dans les Balkans, entendue comme une partie intégrante de l'histoire du quotidien en Europe, se prête encore à diverses interprétations. Les historiens, anthropologues, ethnologues, sociologues et culturologues qui travaillent sur les Balkans sont confrontés à une difficile alternative: la «vie privée» résulte-t-elle d'un processus de civilisation historiquement et géographiquement situé qui aurait débuté pendant la Renaissance en Europe de l'Ouest ${ }^{1}$ ou la "sphère privée " représente-telle une constante des sociétés humaines revêtant des formes diverses en fonction des cultures et des périodes historiques considérées ${ }^{2}$ ? Les rares études disponibles sur la vie quotidienne en Europe du Sud-Est ${ }^{3}$, à l'image de celle d'Evelyne Patlagean consacrée à Byzance aux Xème/ XIème siècles ${ }^{4}$, montrent qu'au Moyen Age déjà, dans les Balkans, l'univers intime de l'individu ne se limitait pas au seul espace de la maison et de la famille comme nous sommes accoutumés à le penser. L'examen, dans une perspective historique ou contemporaine, de la mobilité de travail dans les Balkans soulève des problèmes similaires dans la mesure où elle n'échappe pas à la tendance générale à faire des migrations internationales un objet de débats politiques plus qu'un thème de recherche dont les dynamiques sous-jacentes et les caractéristiques socioculturelles se prêteraient à l'analyse ${ }^{5}$. Rares sont les chercheurs qui tentent de déterminer dans quelle mesure les migrations de travail (temporaires) résultent d'une décision personnelle - une partie de la «sphère privée » et des relations de famille - ou sont le fruit d'une tradition et de modèles de comportement collectifs hérités, que l'on rencontre dans des régions entières. 
2 L'objectif du présent article est d'étudier le phénomène social des migrations temporaires masculines (gurbet/perčalbarstvo) - encore peu connu dans ses dimensions historiques, culturelles et ethnologiques ${ }^{6}$ - en revenant sur les origines de cette tradition dans les Balkans centraux. Seront brièvement présentés ici les résultats d'enquêtes de terrain réalisées entre 2001 et 2005 dans les régions de la péninsule balkanique situées au croisement entre les frontières de trois Etats contemporains, la Bulgarie, la Serbie et la Macédoine, ainsi que dans les montagnes de l'ouest de la Macédoine. Dans les Balkans comme dans le reste de l'Europe de l'Est, cet enjeu a gagné en importance et en visibilité avec l'apparition de nouvelles vagues migratoires de travail en Europe de l'Ouest et en Amérique durant les « décennies de transition » de la fin du siècle dernier. Outre les matériaux rassemblés par l'auteur au cours de ses enquêtes de terrain (mémoires, histoire orale, documents), l'article présentera des sources écrites moins populaires et rarement usitées, à savoir les écrits anciens d'auteurs de Bulgarie, de Serbie et de Macédoine, principalement des historiens et ethnographes locaux, ainsi que des mémoires non publiés de révolutionnaires locaux, à l'image, par exemple, de Simi Sokolov. La migration du travail saisonnière (gurbet/ perčalbarstvo) sera présentée dans ses formes historiques (dans le contexte de la culture traditionnelle et de son quotidien), ainsi qu'à travers les transformations qu'elle a subies au fil du temps.

\section{I - Le phénomène socioculturel du gurbet dans les Balkans}

3 Les migrations de travail transnationales temporaires saisonnières de larges groupes de population vers des régions perçues comme «étrangères » (des migrations limitées à la péninsule balkanique dans la seconde moitié du XIXème siècle, élargies à l'Europe et à l'Amérique au début du XXème siècle) ont traditionnellement reçu l'appellation de gurbetčijstvo ${ }^{7} e t / o u$ de pečalbarstvo dans les langues balkaniques. Dans l'ensemble, ces migrations temporaires de travail (gurbetčijstvo) des dernières décennies de l'Empire ottoman - qui nous sont connues grâce aux sources historiques - sont associées à un large spectre d'activités agricoles et artisanales. Dans l'agriculture, les migrations saisonnières prennent surtout la forme de déplacements de main d'œuvre, pendant la saison des récoltes, depuis les montagnes (des zones caractérisées, selon Fernand Braudel, par l'archaïsme et la pénurie) en direction des riches plaines et des vallées un mouvement que l'on retrouve dans toute l'aire balkanique et méditerranéenne ${ }^{8}$. Combiné à des degrés divers avec le travail agricole, le métier de berger et l'élevage saisonnier (entre les fêtes de la Saint Georges au mois de mai et la Saint Dimitri au mois d'octobre) sont également caractéristiques du XIXème siècle et de la première décennie du XXème siècle. Ces migrations saisonnières agricoles présentent, dans les diverses zones des Balkans, des structures distinctes en termes d'âge et de genre; néanmoins dans leur variante féminine ${ }^{9}$, elles sont exclusivement le fait de jeunes filles ${ }^{10}$. Après leur mariage, les femmes restent traditionnellement auprès de leur famille, dans la maison de leur époux. Les guerres balkaniques de 1912-1913 mettront un terme à ces migrations en bouleversant la distribution du territoire de l'Empire ottoman.

Dans les régions montagneuses du centre et de l'est de la péninsule balkanique, le travail artisanal migrant, masculin et temporaire (gurbetčijstvo) est pratiqué massivement et il est traditionnellement très prestigieux ${ }^{11}$. Cette observation vaut 
particulièrement pour la région historico-culturelle située au cœur des Balkans et connue dans la littérature sous le nom de Šopluk ${ }^{12}$ (un terme qui désigne, dans la littérature ethnographique, la région allant de Niš, sur le territoire de l'actuelle Serbie, jusqu'à Ihtiman, en Bulgarie, et de Vraca, en Bulgarie jusqu'à Kumanovo, en Macédoine); des légendes circulent sur ces hommes « capables de ferrer une puce et de couper en neuf les semelles $»^{13}$. Cependant, dans les Balkans, les flux migratoires d'ouvriers saisonniers (pečalbari) les plus connus sont ceux des montagnes situées à l'ouest de la Macédoine actuelle (la région de Mijak), où l'environnement socio-culturel des villages s'est profondément transformé au cours des siècles sous l'effet des départs temporaires des hommes. Ces régions doivent donc être incluses dans l'étude en tant que base de comparaison possible.

5 Les migrations saisonnières des hommes-pečalbari des Balkans centraux sont bien attestées dans les sources ottomanes et les écrits de diplomates occidentaux ${ }^{14}$, de la seconde moitié du XIXème siècle, dans la décennie postérieure à la guerre de Crimée (1853-1856). Leurs mouvements sont principalement liés à l'exercice d'un artisanat de la construction (djulgerstvo) : l'historien tchèque, Konstantin Jireček, écrit ainsi, en 1899, « du printemps jusque tard dans l'automne » les hommes parcourent la péninsule balkanique «depuis la Serbie et la Valachie [les villages autour du Danube dans l'actuelle Roumanie] jusqu'à Istanbul, à l'Asie mineure et à la Perse $»^{15}$. On rencontre dans la littérature l'hypothèse selon laquelle cette tradition du travail migrant dans le bâtiment plongerait ses racines dans les obligations d'entretien des routes auxquelles une partie de la population masculine locale était astreinte dans l'Empire ottoman. Cette population jouissait d'un statut spécifique, connu sous le nom de voynugan et de dervendji, lié à la logistique de l'armée du Sultan ${ }^{16}$. Il serait plus juste selon moi de rechercher l'origine de la migration saisonnière des hommes de ces régions de montagne dans le déclin de l'élevage des moutons, qui avait été encouragé et organisé par l'administration ottomane afin de satisfaire les besoins de l'armée pendant les premiers siècles de l'Empire. A la différence de la Šumadija en Serbie, par exemple, où le développement économique plus tardif a été lié à l'expansion de l'élevage porcin et au commerce avec l'Empire habsbourgeois, au centre de la péninsule, les registres ottomans ont édifié entre les XVème et XVIIIème siècles un réseau très développé de marchands de bestiaux et d'éleveurs d'agneaux (dželepkešan), principalement des chrétiens, approvisionnant l'Etat, l'armée et la capitale, Istanbul. L'effondrement du système agricole de l'Empire ottoman et la crise socio-économique de la fin du XVIIIème siècle et $d u$ début $d u$ XIXème siècle ont entraîné une diminution des pâturages, la perte du statut privilégié de la population locale et un allongement du cycle de complexité dans les nombreuses familles élargies (zadrugi) dont les membres vivaient plus longtemps ensemble.

6 Ces processus et la généralisation d'une agriculture de type čiflik (çiflik en turc) conduisent l'historienne, Maria Todorova, à défendre l'idée selon laquelle la zadruga, en tant que forme de complexité des foyers/familles, est un phénomène apparu dans les niches écologiques spécifiques des régions de pâturage ou à activités mixtes (élevage et agriculture) en réponse, nouvelle et cyclique, aux particularités du développement de l'Empire ottoman après le XVIIIème siècle. D'après elle, « la fréquence de distribution géographique de la zadruga suit invariablement la courbe des régions montagneuses des Balkans, traversant les frontières ethniques $»^{17}$. Ces conditions socioéconomiques spécifiques de l'Empire ottoman expliquent également, me semble-t-il, la généralisation des migrations temporaires des hommes (perčalbarstvo) du centre des 
Balkans après le premier quart du XIXème siècle. A ces facteurs sont venues s'ajouter, dans le cas de l'ouest de la Macédoine, les incertitudes que faisaient peser, sur l'élevage des agneaux, les attaques incessantes des bandes de voleurs albanais. Plusieurs analystes de la fin du XIXème siècle voient dans cette insécurité la cause de l'accroissement rapide des migrations de travail ${ }^{18}$.

Le « déversement » saisonnier de la population masculine des montagnes allant gagner de l'argent (na pečalba) dans d'autres zones des Balkans a, en retour, contribué à inscrire dans la durée les familles élargies (zadrugite) et à conférer aux réseaux lignagers de ces régions une force et une efficacité proverbiales. La forme traditionnelle d'organisation des groupes de migrants du travail constitue également un facteur important pour comprendre la préservation et l'importance accordée à la structure familiale/de lignage dans la vie villageoise : ces groupes étaient formés sur un principe lignager et/ou de solidarité villageoise-locale et ils n'ont connu aucune réglementation écrite (de type guilde), pas même au début du XXème siècle; ils ont suivi les normes de la pratique coutumière. Ce fait, tout comme le manque de données statistiques ${ }^{19}$ concernant l'ampleur des migrations saisonnières depuis la Bulgarie, la Serbie et la Macédoine ${ }^{20}$, explique la stratégie de recherche retenue ici: une reconstruction ethnographique reposant exclusivement sur des sources narratives, c'est-à-dire sur des récits oraux réunis dans des mémoires ou collectés par l'auteur, dans un travail d'histoire orale. Les premières tentatives de réglementation centralisée de l'artisanat alors traditionnellement le plus populaire parmi les migrants temporaires, la construction, datent du XIXème siècle. A partir des années 1890, à Crna Trava en Serbie, sont ainsi organisés, l'hiver, des cours trimestriels spécialisés de maçonnerie ${ }^{21}$ et, en 1903, une école du bâtiment est ouverte à Trăn, en Bulgarie. Réputée dans tout le pays, elle a continué à fonctionner jusqu'à aujourd'hui.

\section{II - Sur les routes du gurbet}

8 Les directions, les destinations et le caractère du travail saisonnier comme des migrations temporaires changent à plusieurs reprises au cours du XIXème siècle et dans les premières décennies du XXème siècle, sous l'effet de l'histoire tumultueuse de la péninsule. Au cours des cent trente dernières années, ces régions ont plusieurs fois changé d'appartenance étatique - ce qui, dans les Balkans, équivaut le plus souvent à un changement d'identité nationale, particulièrement dans les régions frontalières ${ }^{22}$. Avant l'accession de la Bulgarie à une indépendance de fait en 1878, les artisans du bâtiment se dirigeaient prioritairement vers la capitale de l'Empire ottoman, Istanbul. Les migrants saisonniers partaient au moment des grandes fêtes du printemps Mladenci (la Fête des quarante martyrs), Džurdžovdăn (la Saint Georges) - ou, plus souvent, au début du carême de Pâques. Au moment de la Saint Constantin et Hélène, en mai, ils étaient déjà "au travail» ( $u$ rabotu») $)^{23}$. La Valachie (aujourd'hui en Roumanie) et la Serbie, alors libres, représentaient d'autres destinations privilégiées. Les migrants (pečalbari) se rendant en Valachie se rassemblaient à Godeč (aujourd'hui en Bulgarie), passaient par le col de Petrohan, la ville de Lom, le port de Turnu Severin et de Cetatea sur la rive roumaine du Danube, avant d'atteindre les villages de l'actuel sud de la Roumanie. Là-bas, ils ont construit des «bienici » célèbres - des maisons en terre battue particulièrement appréciées par la population valaque locale ${ }^{24}$. Dans plusieurs villages du sud-ouest de la Serbie et du centre-ouest de la Bulgarie, à la fin du 
XIXème siècle, la population masculine parlait couramment le roumain, une langue apprise à l'occasion des migrations saisonnières (u pečalbu) en Valachie ${ }^{25}$.

Pour ceux qui se rendaient en Serbie, avant 1878 les points de rassemblement étaient Smederevo, Paračin, Jagodina et Čjuprija. De là, les groupes de migrants saisonniers employés dans le bâtiment se dispersaient à travers toute la Šumadija. Dans la région de Trăn (centre-ouest de la Bulgarie) les hommes qui pratiquaient l'art de la construction dans la Serbie souveraine étaient connus sous le nom de šumadijci pour les distinguer des stamboldžiite, que le gurbet portait vers Istanbul et ses environs ${ }^{26}$. Certains des premiers grands entrepreneurs du bâtiment en Serbie et dans sa capitale, Belgrade, étaient originaires des régions de Crna Trava (aujourd'hui en Serbie) et de Trăn (aujourd'hui en Bulgarie) ${ }^{27}$.

Plusieurs de ces maîtres-maçons et de leurs groupes d'ouvriers ont pris activement part aux luttes pour l'indépendance nationale des populations locales au XIXème siècle : lors de la prise de la forteresse de Belgrade en 1862, des soulèvements de la région de Sofia (Šopskoto) en 1877 ou encore de la région de Kresno-Razlog en 1878. A Čuprija précisément, en 1862, peu après la formation de la Première légion bulgare à Belgrade, à la demande de Georgi Rakovski, le célèbre architecte Grozdan Nasalevski a formé trois détachements de volontaires bulgares issus du milieu des ouvriers du bâtiment originaires de Trăn, qui ont pris part à la guerre serbo-turque ${ }^{28}$. Certains de leurs leaders (pečalbarski tajfi) ont obtenu des grades militaires dans les armées russe et serbe; ils ont rejoint en tant que volontaires les troupes du général Černajev au moment de la guerre serbo-turque de 1876-1877, puis les volontaires bulgares lors de la guerre russo-turque de1877-1878 au terme de laquelle la Bulgarie a acquis l'indépendance. Les détachements d'un autre habitant réputé de Trăn, Simo Sokolov, ont participé, aux côtés des armées russe et serbe, à la libération consécutive des régions de Trăn (aujourd'hui en Bulgarie), Vranja (aujourd'hui en Serbie), Kriva Palanka et Kratovo (au nord-est de l'actuelle Macédoine) ${ }^{29}$. Ils ont également pris part au soulèvement de Kresna-Razlog, qui a éclaté après le Congrès de Berlin de juin-juillet 1878, dans les régions bulgares restées dans l'Empire ottoman.

11 Après la Libération de la Bulgarie, en 1878, la nouvelle capitale, Sofia, devient rapidement un centre attractif pour les bâtisseurs-pečalbari des régions de Trăn et de Caribrod en Bulgarie, de Crna Trava, Vranja et Pirot en Serbie et du nord-est de la Macédoine restées à l'intérieur des frontières ottomanes. D'après les données approximatives de Jelenko Petrović, pendant la dernière décennie du XIXème siècle et jusqu'en 1912, quelque 8000 personnes se rendaient chaque année à Sofia depuis la Serbie, dont 2000 environ de la seule région de Pirot $^{30}$. Dans plusieurs villages du sudest de la Serbie, jusqu'à un quart de la population masculine allait ainsi travailler en Bulgarie, le plus souvent à Sofia. De l'importance de ces migrations transfrontalières, nous pouvons seulement juger à partir d'un fait intéressant : dans la première décennie du XXème siècle, les monnaies bulgare et serbe étaient toutes deux, et à parts égales, en circulation à Pirot (Serbie) ${ }^{31}$. Cette situation a suscité une ingérence administrative active des pouvoirs serbes dans la région. Avec Cvetko Radokov, originaire de Trăn, le grand homme politique, Nikola Pašić, s'est également établi en tant qu'entrepreneur dans le bâtiment à Sofia, pendant les années de son bannissement forcé après le soulèvement de Zajčarska contre le roi Milan. Leur activité dans la construction a servi à dissimuler la préparation d'un soulèvement contre le roi en Serbie ${ }^{32}$. 

connus à Sofia à la fin du XIXèle siècle et au début du XXème siècle étaient originaires de Macédoine. A juste titre, le chemin qui, passant par Kriva Palanka, conduisait depuis la Macédoine vers Sofia était appelé par les contemporains «le chemin des hommes partis faire de l'argent » (pečalbarski drum), parce que, tous les printemps, plus de 10000 hommes de Macédoine parcouraient cette route pour se rendre dans la capitale de la Bulgarie libérée ${ }^{33}$. Leur nombre, pour les seuls villages autour de Kriva Palanka, est estimé à plus de trois mille ${ }^{34}$. En dehors de la construction, les migrants et les employés temporaires de Macédoine ont développé dans la capitale bulgare d'autres artisanats, comme la boulangerie, la crèmerie, la pâtisserie et le commerce. Les migrants temporaires de Macédoine occidentale (des régions de Tetovo, Debăr, Kičevo, Bitola et Kostur/Castoria), de Salonique et d'Istanbul avaient le même type d'activités ${ }^{35}$. Les guerres balkaniques de 1912-1913 et les nouvelles frontières politiques des Balkans signent la fin de ces migrations temporaires en fermant les routes, en direction du sud, traditionnellement suivies par les travailleurs saisonniers.

De nouvelles destinations les conduisent alors depuis la Macédoine occidentale principalement vers Belgrade, depuis la Macédoine orientale en direction de Sofia ${ }^{36}$ et, pour la population albanaise, vers l'Albanie devenue indépendante. Au début des guerres balkaniques et pendant la première Guerre mondiale, un grand nombre de ces travailleurs temporaires des Balkans centraux émigre vers l'Amérique afin d'échapper à la conscription. A partir de 1900, l'Amérique se transforme en une terre d'immigration attractive pour la main d'œuvre sans emploi de la région, d'abord celle de Macédoine ${ }^{37}$, puis, plus tard, de Bulgarie et de Serbie. Une partie de ces «Américains » est revenue dans les années 1920 ; toutefois nombreux sont ceux qui sont restés. Une autre frange des pečalbari de Macédoine prend part aux guerres balkaniques avec l'espoir de libérer leur terre d'origine du pouvoir ottoman ${ }^{38}$.

\section{III - Les cultures au quotidien : vivre en l'absence des hommes}

Organisés selon un principe lignager ou territorial, les groupes d'hommes migrant temporairement (pečalbarski tajfi) développent leur sous-culture spécifique à l'intérieur des grandes villes qui les accueillent, à Istanbul, Thessalonique, Belgrade et Sofia. Les hommes ont leurs lieux de rencontre et de discussion comme le célèbre hôtel Znepole à Sofia, où se retrouvent les ouvriers du bâtiment originaires de Trăn ou l'auberge Razlog, point de rencontre des migrants de Macédoine. Belgrade compte quelques kafani «macédoniens » à Čubura, dont la clientèle est issue de Macédoine occidentale ${ }^{39}$. Les particularités de leur dialecte font de celui-ci leur marqueur linguistique (et une langue originale «secrète ») en Bulgarie comme en Serbie ${ }^{40}$. Les groupes migrants des Balkans centraux sont acceptés par la population locale des deux côtés de la frontière comme une communauté spécifique: les bâtisseurs de Crna Trava en Serbie tout comme ceux de la région de Trăn en Bulgarie sont traditionnellement appelés Kărkavci ${ }^{41}$ et leurs groupes à déplacement saisonnier sont comparés aux bandes d'oiseaux migrateurs (les grues). Ces communautés d'artisans restent traditionnellement repliées sur leur sous-culture : la présence, dans leurs groupes, d'ouvriers issus d'autres régions constitue une rare exception jusqu'au milieu du XXème siècle. Les maçons de Kriva Palanka (aujourd'hui en Macédoine) racontent encore que, dans les années 1930-1940, ils préféraient 
travailler à Skopje avec des ouvriers bulgares de Bosilegrad (aujourd'hui en Serbie) plutôt qu'avec des bâtisseurs de Macédoine occidentale (de Vevčani), qui étaient d'une autre « école».

Les voyages annuels des hommes des Balkans centraux pratiquant le gurbet ont suscité l'émergence, à travers les années, d'un système festif et rituel et d'un folklore spécifiques, avec des chansons du type «je vais à l'étranger, je quitte le paradis $»^{42}-$ “Tugjina idem, ostavjam raj!". En parallèle avec l'allongement du cycle de complexité des lignées et des familles élargies, s'observe un regroupement des fêtes familiales et lignagères les plus importantes (de type slava/služba/svetec) pendant l'automne et l'hiver du calendrier folkorique festif - des fêtes de la Saint Petka /Saint Parascève et de la Saint Dimitri au mois d'octobre jusqu'à la fête de Saint Athanase en janvier ${ }^{43}$. Mitrovden (la fête de la Saint Dimitri), Rangelovden (le jour de l'Archange Michel), Nikulden (la fête de Saint Nicolas) et Božik (la naissance du Christ) deviennent les centres sacrés du cycle du calendrier des fêtes et des rituels de famille ${ }^{44}$. Dans les villages de Macédoine occidentale, qui ont connu une longue tradition de pečalbari, on observe une créativité intéressante dans le processus rituel de célébration des fêtes dans l'esprit de la «tradition inventée $»^{45}$ - à Vevčani, la fête Spassovden (l'ascension du Christ) est célébrée trois fois, en mai, en septembre et en janvier. La fête du jeudi avant le jour de la Saint Athanase (31 janvier) est appelée le "Spasovden pečalbar", parce que les hommes rentrés au village après avoir travaillé à l'étranger visitent la chapelle construite à flanc de montagne pour et par des travailleurs migrants ${ }^{46}$.

Dans les régions pečalbari des Balkans étudiées, toute la destinée de l'individu est prédéterminée par cette stratégie essentielle du travail temporaire/saisonnier. Chez les nouveaux-nés, le rituel traditionnel du baptême comprend une série de bénédictions et de symboles spécifiques qui visent à assurer au garçon, de manière magique, un destin de bon ouvrier-maître maçon. En Macédoine, le premier voyage des garçons en dehors de leur terre d'origine («le premier solunče» à Galičnik) s'accompagne de rituels particuliers et de bénédictions en vue de rapporter un " grand gain ». Dans les régions montagnardes des Balkans centraux et de Macédoine occidentale ${ }^{47}$, la tradition populaire locale a élaboré un solide complexe rituel gurbet, lié au départ et au retour des groupes de migrants temporaires. Les femmes expédient leur mari et leurs fils loin au-delà des limites du village jusqu'à un endroit traditionnellement défini comme marquant la frontière de la région (Dăsčan kladănăc pour Znepole, Plači most pour Želino, la région de Tetovo et de Kičevo, Plači kruša pour Lazaropole et Vevčani, etc.), où se rassemblent les groupes de travailleurs saisonniers. Les appellations de ces toponymes pečalbari sont le plus souvent liées à la plainte et elles évoquent des scènes pathétiques de séparation familiale (temporaire). Telle est l'origine du vieux nom de kurbet planina (la montagne kurbet) qui sépare la région de Nišava de la vallée de la Morava ${ }^{48}$ dans la partie centrale des Balkans.

17 L'absence des hommes-pečalbari de leurs maisons pendant la majeure partie de l'année conduit à des transformations dans tous les rituels fondamentaux du cycle de vie. Dans les régions de montagne des Balkans centraux, les mariages sont célébrés l'hiver, lorsque les hommes-constructeurs sont de retour chez eux. Chez les habitants de la région de Mijak, en Macédoine occidentale, les mariages ont lieu seulement une fois par an, le jour de la fête du Saint patron du village (Petrovden à Galičnik ${ }^{49}$, Ilinden à Lazaropole), lorsque les jeunes reviennent dans leur région natale. Si les jeunes fiancés (verenici) ne parviennent pas à s'unir ce jour-là, ils doivent patienter une année entière 
jusqu'à la prochaine fête. La seule variante de "réserve " autorisée par la tradition aurait été la fête de l'Assumption. Jusqu'au milieu du XXème siècle, ces régions de montagne sont strictement endogamiques. Dans quelques villages de Macédoine occidentale, cette endogamie est même interne au village : en vue de leurs noces, les jeunes hommes reviennent chercher des jeunes filles à marier dans leur région de naissance, en principe seulement parmi les "leurs » (d'un point de vue villageoisrégional). D'après les personnes interviewées, aujourd'hui encore les jeunes femmes se marient le plus souvent pendant l'été, quand les descendants des pečalbari d'autrefois et des gastarbeiter d'Amérique et d'Australie rentrent chez eux y chercher des épouses « convenables».

18 La tradition locale des migrations de travail temporaires a également donné naissance à un rituel complexe pour les enterrements des ouvriers saisonniers morts à l'étranger. Ces derniers sont symboliquement enterrés et pleurés dans leur village d'origine. Cette pratique rituelle est fréquente dans les Balkans ; mais, dans le village de Mešeišta (de la région de Struga, à l'ouest de la Macédoine), au cours de mes enquêtes de terrain en 2005 , je suis tombé sur une forme accomplie de transformation de cette pratique. A la fin du XIXème siècle, à côté de l'église du village avait été creusée une tombe vide, dite jabandžijski, sur laquelle les proches pleuraient les hommes-ouvriers temporaires morts et enterrés quelque part à l'étranger. Restaurée à la fin du XXème siècle, cette tombe jabandžijski vide se dresse aujourd'hui, tel un monument au «pečalbar inconnu » des Balkans.

\section{IV - Le temps des Gastarbeiter et les transformations des modèles traditionnels}

Dans les pays de l'ancienne Yougoslavie, ce modèle traditionnel de migrations de travail saisonnières établi depuis des siècles s'est transformé au début des années 1970. Durant les dernières décennies du XXème siècle, les migrants temporaires des régions de Serbie et de Macédoine que j'étudie ont commencé à émigrer définitivement avec leurs familles en Europe occidentale et en Amérique (Etats-Unis et Canada), en Australie et en Nouvelle Zélande. Ce processus de transformation de migrants temporaires en émigrants était une conséquence de la nouvelle politique migratoire adoptée par plusieurs pays européens, au premier chef l'Allemagne (après 1972), qui facilitait l'immigration légale. Invités à travailler légalement pendant une période déterminée dans plusieurs pays d'Europe occidentale en quête de main d'œuvre agricole, les Gastarbeiter des Balkans ont bientôt fait venir leurs familles avec eux et se sont définitivement établis dans le pays qui les avait accueillis. L'Allemagne a "partagé » son modèle de migrations de travail temporaires, ainsi que l'appellation Gastarbeiter, avec les autres Etats d'Europe de l'ouest. Il en est résulté un bouleversement complet dans le modèle d'organisation des familles (temporairement) séparées dans les régions que j'étudie, en particulier en Macédoine occidentale. Le modèle du gurbet où les familles restent sur le lieu de naissance pendant que les hommes " gagnent de l'argent » à l'étranger - un argent qu'ils envoient à leurs proches et dépensent "à la maison » - a évolué vers un modèle pečalbar de culture Gastarbeiter. La majorité des Gastarbeiter, invités à venir travailler dans les pays d'Europe occidentale en tant que main d'œuvre légale (principalement en Allemagne de l'ouest), s'installent définitivement avec leurs familles dans les pays d'accueil. Et bien qu'une partie 
significative d'entre eux retournent dans leur pays d'ex-Yougoslavie au moment de leur retraite, leurs enfants, nés en Europe occidentale, restent dans la plupart des cas dans leur nouvelle patrie. Le modèle de migration du travail depuis les Balkans de type Gastarbeiter transforme aussi bien la situation sociale des pays d'émigration (avec un apport économique significatif pour les Républiques d'ex-Yougoslavie), que les modes d'identification des fils et filles nés en Europe occidentale ${ }^{50}$.

De nombreux villages ont été désertés pendant les décennies du socialisme. Mais la nostalgie de «la terre d'origine» est demeurée et une partie de ces Gastarbeiter reviennent du monde entier dans leur village des Balkans passer leurs derniers jours «chez eux, auprès des leurs». Au sud-ouest de la Macédoine, nombreux sont les exemples de Gastarbeiter qui, bien qu'ils aient été naturalisés dans leur «nouvelle patrie ", construisent une nouvelle maison dans leur village ou achètent à leurs enfants un appartement dans le principal centre urbain de leur région d'origine (Struga, Ohrid). Dans plusieurs villages de la région de Debar, ce sont eux qui financent la reconstruction des anciens lieux de culte orthodoxes, la construction de nouveaux lieux de culte et de chapelles, ainsi que celle de nouveaux bâtiments publics. Les exemples ne sont pas rares en Macédoine de villages qui furent pendant des décennies entièrement abandonnés et dans lesquels les populations locales reviennent chaque année pour la fête du Saint patron, afin de pratiquer collectivement le sacrifice du kurban et de partager un repas "afin que le village survive ${ }^{51}$. L'effondrement de l'ancienne Fédération yougoslave, les guerres et conflits ethniques qui l'ont accompagné, l'émergence de nouveaux Etats indépendants dans les Balkans occidentaux et l'introduction de nouvelles frontières étatiques difficiles à franchir ont, à bien des égards, transformé ce modèle traditionnel pečalbar/Gastarbeiter, faisant apparaître, parmi les représentants des nouvelles générations de nouvelles stratégies de survie et de nouvelles attentes. Comment vont se développer les relations entre les diasporas de Gastarbeiter et les colonies de pečalbari dans le Nouveau monde (y compris en Australie et en Nouvelle-Zélande) et les communautés locales dans leurs régions d'origine des Balkans, l'avenir nous le dira. Pour le moment, nous pouvons seulement observer des processus d'identification complexes et pluriels chez les représentants de la nouvelle génération d'émigrés, dont la plupart sont nés en dehors des Balkans. D'un côté, ils font partie intégrante de la diaspora "balkanique », ancienne ou plus récente, dans les pays d'acceuil ; de l'autre, le retour dans les Balkans, au village, chez leurs parents âgés, est toujours perçu comme un moment de fête.

21 En Bulgarie, les années de la transition ont vu se développer des formes de migrations du travail qui ont reproduit certains des traits de l'ancien modèle transfrontalier $d u$ gurbet, tout en présentant de nouvelles caractéristiques, qui nous sont connues grâce à la culture Gastarbeiter des migrants temporaires d'ex-Yougoslavie. Dans un premier temps, des hommes (mais aussi de plus en plus souvent des femmes ces dernières années) quittent leur lieu de naissance en petits groupes pour aller travailler en Europe occidentale, d'où ils envoient de l'argent pour aider leur famille restée en Bulgarie à subvenir à ses besoins. Même lorsqu'ils font venir leur famille ultérieurement, ils continuent à aspirer à rentrer dans leur patrie, y compris pour y montrer leur réussite obtenue grâce « au gurbet ». L'étude de ces nouvelles migrations temporaires de travail (pečalbari) dépasse toutefois le cadre du présent article, tout comme celle des perspectives qui leur ont été ouvertes par la nouvelle adhésion de la Bulgarie à l'Union européenne ${ }^{52}$. 
Pour conclure, les migrations de travail temporaire des hommes des régions montagneuses des Balkans centraux et de Macédoine occidentale ont suscité des transformations spécifiques dans le modèle socio-culturel traditionnel de la population locale, aussi bien dans l'organisation sociale que dans les modèles familiaux et les stratégies maritales, liées à l'allongement du cycle de complexité des lignées familiales et aux particularités des rôles hommes-femmes traditionnels, ainsi que dans les rituels du cycle de vie et du cycle calendaire. Les guerres balkaniques du début du XXème siècle ont entraîné un changement dans les directions de ces migrations temporaires (plus que dans leur nature). La nouvelle politique migratoire adoptée par certains pays d'Europe occidentale au début des années 1970 a, par ailleurs, radicalement modifié le caractère des relations sociales dans les régions pečalbari/Gastarbeiter des Balkans, en particulier dans les pays de l'ancienne Yougoslavie (y compris dans l'actuelle République de Macédoine). La pratique séculaire des hommes qui partaient « là-bas ", à "l'étranger » afin de subvenir aux besoins des familles «ici ", dans les Balkans, s'est transformée en une possibilité d'émigration légale avec réunion familiale dans les pays d'accueil entre les années 1970 et la fin du socialisme. En Bulgarie, le modèle de migration du travail d'ampleur limitée, contrôlée par l'Etat en direction des pays de l'ancien Bloc soviétique a également subi des transformations majeures après 1990, avec une accélération des flux migratoires illégaux temporaires (jusqu'à trois mois) en direction des pays de l'Union européenne, une mobilité transfrontalière renforcée et une féminisation du gurbet.

Les transformations intervenues au cours de la dernière décennie du XXème siècle dans la carte socio-économique de la péninsule balkanique ont fait passer certains Etats, comme la Grèce, du statut de pays d'émigration à celui de pays d'immigration, accueillant des travailleurs migrants saisonniers et temporaires. Dans le même temps, les traditions pečalbar et les représentations sociales spécifiques liées au gurbet démontrent une certaine résilience dans plusieurs régions des Balkans d'où sont issues les nouvelles vagues de gurbetčii (pečalbari). Souvent, ces derniers, sous l'effet des conditions nouvelles qui prévalent dans la région, s'installent définitivement dans le pays qui les a accueillis, où ils deviennent des immigrants. La décision des pečalbari d'émigrer définitivement ou de continuer à chercher à revenir dans leur région d'origine dépendra dans une large mesure du développement socio-économique des Balkans et de l'avenir géopolitique des pays de la région. Des études ultérieures des mobiltiés de travail et des migrations depuis les Balkans détermineront dans quelle mesure la formule folklorique traditionnnelle «Où que j'aille, je reviendrai ", que l'on rencontre dans le folkore pečalbar de plusieurs peuples balkaniques, continuera à s'appliquer au modèle de comportement social connu sous le nom de gurbet.

\section{BIBLIOGRAPHIE}

Ariès P., Duby G., 1985 - Histoire de la vie privée. Vol. 1-5, Paris : Seuil. 
Bobčev S., 1908 - Sbornik na bălgarski juridičeski običai [Recueil des coutumes juridiques bulgares], vol. 2, Sofia.

Braudel F., 1998 - Sredizemno more i sredizemnomorsijat svjat po vremeto na Filip II, [La Méditerranée et le monde méditerranéen a l'époque de Philippe II], kn.1, Sofia.

Brunnbauer U., 2004 - « Environment, Markets and the State: Human Adaptation in the Balkan Mountains, $19^{\text {th }}-$ early $20^{\text {th }}$ Century $»$, Ethnologia Balkanica, 8, p.129-154.

Cvijić J., 1906 - ЦВијић J., Osnove za geografiju I geologiju Makedonije i Stara Srbije, Beograd.

Cvijić J., 1912, Naselja srpskih zemalja, Beograd.

Cvijić J., 1922 - ЦВијић J., Balkansko poluostrvo i južnoslovenske semlje, кnj. prva, Beograd.

Cvijić J., 1931 - ЦВијић J., Balkansko poluostrvo i južnoslovenske semlje, кnj. druga, Beograd.

Duerr H. P., 1988 - Nacktheit und Schamm. Der Mythos vom Zivilisationsprozess, Frankfurt am Main.

Natan Ž., Berov L., Hadžinikolov V., 1969 - Ikonomika na Bălgarija [Economie de la Bulgarie], т. 1. Ikonomikata na Bălgarija do socialističeskata revoljucija [Economie de la Bulgarie jusqu'à la révolution socialiste], Sofia.

Elias N., 1978 - Über den Prozess der Zivilisation: Soziogenetische und psychogeneitsche Untersuchungen, Frankfurt am Main, Bd. 1-2.

Gerov N., 1895 - Rečnik na Bălgarskija ezik, [Dictionnaire de la langue bulgare] vol. 1, Plovdiv.

Gerov N., 1908 - Dopălnenie na bălgarski rečnik ot N. Gerov [Compléments au dictionnaire de la langue bulgare], Plovdiv.

Hobsbawm E., 1983 - « Introduction: Inventing Traditions », in: Hobsbawm E., Ranger T.(eds.), The Invention of Tradition, Cambridge University Press, p.1-14.

Hristov P., 2001 - «Ahnenkult in Westbulgarien : das Fest des Schutzheiligen », in : Brunnbauer, U. und Kaser K. (Hg.), Vom Nutzen der Verwandten. Soziale Netzwerke in Bulgarien (19. und 20. Jahrhundert), Böhlau, Wien, p.187-199.

Hristov P., 2002 - « The Use of Holidays for Propaganda Purpose. The "Serbian" Slava and/or the "Bulgarian" Sabor ", Ethnologia Balkanica, 6, p.69-80.

Hristov P., 2003 - « Nikola Pasič i njegovo delovanje u emigraciji u Bugarskoj”, Razvitak, nº. 213-214, Zaječar, 93, 113-120.

Hristov P., 2004-A - « Granicite na "Šopluka" i/ili šopi bez granici » [Les frontières du « Šopluk » et/ou les Šops sans frontières], in : Skrivene manjine na Balkanu, Beograd, p.67-82.

Hristov P., 2004-B - « Praznikot na pustoto selo (Sliki ot ritualniot proces vo R. Makedonija i R. Bugarija) » [La fête du village quitté (Images du processus rituel en République de Macédoine et en République de Bulgarie)], MAkedonski folklor, 62, Skopje, 2004, p.105-122.

Hristov P., 2005 - « The Market and the Piazza for Hired Hand in Sofia as Places to Exchange Cultural Stereotypes ", Ethnologia Balkanica, 9, p.82-90.

Irechek K., 1976 - Pătuvanija po Bălgarija [Voyages en Bulgarie], Sofia.

Irechek K., 1978 - Istorija na Bălgarite [Histoire des Bulgares], Sofia.

Karamihova M., 2003 - Da živeeš tam, da se sănuvaš tuk. Emigracionni procesi v načaloto na XXI vek [Vivre là-bas, se rêver ici. Les processus émigratoires au début du XXème siècle], Sofia. 
Karovski L., 1979 - Makedonski pečalbarski narodni pesni [Chansons populaires pečalbar macédoniennes], Skopje.

Kaser K., 1994 - « Privatni život u Jugoistočnoj Europi » [La vie privée en Europe du Sud-Est], OTIVM 2(1-2), Zagreb, p.48-55.

Kearney M., 1997 - «Migration», in : Barfield T. (ed.), The Dictionary of Anthropology, Blackwell, Oxford, p.322-324.

Kjushkosky A., 1998 - Pečalbarstvoto vo Vevčani [Les pratiques pečalbar à Vevčani], Bitola.

Koneska E., 1992 - « Makedonci vo Istanbul » [Macédoniens à Istanbul], Etnolog, 1, Skopje, p.64-72.

Mironova-Panova S., 1971 - Trănskijat kraj [La région de Trăn], Sofia.

Mihov N. V., 1943 - Prinosi kăm tărnovskata istorija na Bălgarija, vol. II. Avstrijski konsulski dokladi [Contributions à l'histoire de la Bulgarie de Tărnovo. Rapports consulaires autrichiens], T. 1, Sofia.

Nikolić V., 1910 - николић в., «Iz Lužnice i NiǎveE», Srpski etnografski zbornik, kn.. 16, Beograd. Nikolić R., 1912 - николић Р., « Krajište i Vlasina », Srpski etnografski zbornik, kn. 18, Beograd.

Palairet M., 1987 - « The Migrant Workers of the Balkans and Their Villages (18 $8^{\text {th }}$ Century - World War II) », in: Roth K. (Hg.), Handwerk in Mittel- und Südosteuropa, München : SüdosteuropaGesellschaft, p.23-46.

Petrichev L., 1940-A - « Trănskite dobrovolci v Srăbsko-turskata vojna - 1876 godina » [Les volontaires de Trăn dans la guerre serbo-turque en 1876], Trănski kraj, Sofia, p.140-162.

Petrichev L., 1940-B - « Šopskoto vǎstanie prez 1877 godina na trănski kraj » [Les soulèvement šop en 1877 dans la région de Trăn], Trănski kraj, Sofia, p.163-171.

Petrov G., 1896 - MAteriali za izučavaneto na Makedonjia [Matériaux pour l'étude de la Macédoine], Sofia.

Petrov G., 1909 - « Emigranskoto dviženie za Amerika v Makedonija » [Le mouvement d'émigration vers l'Amérique en Macédoine], Kulturno edinstvo, Sofia, p.3-6.

Petrović J., 1920 - ПЕтРовић J., Pečalbari, naročito iz okoline Pirota, Beograd.

Pesheva R., 1960 - «Edin starinen semeen praznik. Praznuvane na svetec v Severozapadnata i Zapadna Bălgarija » [Une veille fête familiale. La célébration d'un saint dans la Bulgazrie du NordOuest et de l'Ouest] , Ezikovedski-etnografski izseldvanija v pamet na akad. Romanski [Etudes ethnographiques et linguistiques en souvenir de l'Académicien Romanski], Sofia, p.731-754.

Resova M., 1955 - « Običai na Mijacite-gurbetčij ot Mala Reka » [Les coutumes des personnes pratiquant le gurbet originaires de la région de Mijak, de Mala Reka], Vesnik na Muzejskokonzervatorskoto društvo na NRM, 1, Skopje, p.7-14.

Samardzić R., M. Djordjević (eds.) 1989 - Migrations in Balkan History, Belgrade.

Spirovska L., 1971 - « Za nekoj mijački pečalbarski običai pocebno ot Galičnik » [De quelques coutumes pečalbar de la région de Mijak, en particulier de Galičnik], Bitorski naučno-kulturni sobiri I, Gostivar, p.257-263.

Todorova M., 2002 - Balkan Family Structure and the European Pattern, Washington.

Valchinova G., 1999 - « Znepolski poxvali ». Lokalna religija i identičnost v Zapadna Bălgarija [Religion locale et identité en Bulgarie occidentale], Sofia. 


\section{NOTES}

1. Elias (Norbert), Über den Prozess der Zivilisation: Soziogenetische und psychogeneitsche Untersuchungen, Bern: Francke Verlag, 1969, Bd. 1-2.

2. Duerr (Hans Peter), Nacktheit, Schamm, Der Mythos vom Zivilisationsprozess, Frankfurt am Main: Suhrkamp Verlag, 1988; pour une discussion de cet argument, voir Kaser (Kaser), «Privatni život u Jugoistočnoj Europi » [La vie privée en Europe du Sud-Est], OTIVM [Zagreb], 2(1-2), 1994, p.48-55.

3. Ariès (Philippe), Duby (Georges), Histoire de la vie privée, vol. 1-5, Paris: Seuil, 1999.

4. Patlagean (Evelyne), "Byzance Xème-XIème siècle", in: Brown (Peter), Patlagean (Evelyne), Rouche (Michel), Thébert (Yvon), Veyne (Paul), De l'Empire romain à l'An mil. Tome 1 de Histoire de la vie privée, Paris : Seuil, 1985, p.531-618.

5. Sur ce point, voir Kearney (M.), « Migration », in : Barfield (Thomas), ed., The Dictionary of Anthropology, Oxford: Blackwell, 1997, p.322-324.

6. Au sein de la très vaste littérature consacrée aux migrations du travail, les mobilités d'Europe du Sud-Est et, plus concrètement, des Balkans n'ont pas occupé une place très significative. Voir, par exemple, Palairet (Michael), «The Migrant Workers of the Balkans and Their Villages (18 ${ }^{\text {th }}$ Century - World War II) ", in Roth (Klaus), Handwerk in Mittel- und Südosteuropa, München: Südosteuropa-Gesellschaft, 1987, p.23-46; Samardzić (Radovan), Djordjević (Mita), eds, Migrations in Balkan History, Belgrade: Srpska akademija nauka i umetnosti, 1989; Puskás (Julianna), ed., Overseas Migration from East Central and Southeastern Europe: 1880-1940, Budapest: Akadémiai Kiadó, 1990; Beer (Mathias), (Hg.), Migration nach Ost- und Südosteeuropa vom 18. bis zum Beginn des 19. Jahrhunderts: Ursachen - Formen - Verlauf - Ergebnis, Stuttgart: Thorbecke, 1999; Brunnbauer (Ulf), «Environment, Markets and the State: Human Adaptation in the Balkan Mountains, $19^{\text {th }}$ - early $20^{\text {th }}$ Century»,Ethnologia Balkanica, 8, 2004, p.129-154; Hristov (Petko), "The Market and the Piazza for Hired Hand in Sofia as Places to Exchange Cultural Stereotypes »,Ethnologia Balkanica, 9, 2005, p.82-90.

7. L'appellation vient du turco-arabe, gurbet, qui signifie « un travail en dehors de son lieu de naissance "; de là, fin XIXème, d'après le dictionnaire de N. Gerov, en langue bulgare, "gurbetuvam» signifiait «aller à l'étranger" (voir Gerov (Najden), Rečnik na bălgarski ezik [Dictionnaire de la langue bulgare], Plovdiv: Pečatnica Săglasie, première partie, p.26), et " gurbetčija », " étranger » (cf. voir Gerov (Najden), Dopălnenie na bălgarskija rečnik ot N. Gerov [Complément au dictionnaire bulgare de N. Gerov], Plovdiv : Pečatnica Trud, 1908, p.83).

8. Braudel (Fernand), Sredizemno more i sredizemnomorsijat svjat po vremeto na Filip II [La Méditerranée et le monde méditerranéen à l'époque de Philippe II], kn.1, Sofia, 1998, p.30, p. 40-43, p.51-53.

9. Voir l'expression bulgare « descendre vers la Roumélie » (slizaneto na Romănja) qui signifiait «partir pour la moisson».

10. Hristov (Petar), « The Market and the Piazza for Hired Hand in Sofia as Places to Exchange Cultural Stereotypes ", Ethnologia Balkanica, 9, 2005, p.87-88.

11. Bobchev (S.), Sbornik na bǎlgarski juridičeski običai [Recueil des coutumes juridiques bulgares], vol. 2, Sofia, 1908, p.107; Petrović (J.), Pečalbari, naročito iz okoline Pirota, Beograd, 1920, p.18; Cvijić (Jovan), Balkansko poluostrvo i južnoslovenske zemlje, Knj. druga, Beograd, 1931, p.134.

12. Hristov (Petar), «Granicite na "Šopluka" i/ili šopi bez granici » [Les frontières du "Šopluk » et/ou les Šops sans frontières], in Skrivene manjine na Balkanu [Les minorités cachées des Balkans], Beograd, 2004, p.67-82.

13. Cvijić (Jovan), Osnove za geografiju i geologiju Makedonije i Stare Srbije [Les bases de la géographie et de la géologie de la Macédoine et de la Vieille Serbie], Beograd, 1906, p.194. 
14. Sur les informations des consuls autrichiens, voir Mihov (Nikola Vasilev), Prinosi kăm tărnovskata istorija na Bălgarija, vol. II. Avstrijski konsulski dokladi [Contributions à l'histoire de la Bulgarie de Tărnovo. Rapports consulaires autrichiens], T. 1, Sofia, 1943, p.331-332.

15. Concernant la Belgrade et toute la Šumadija, voir Irechek (Konstantin), Pătuvanija po Bălgarija [Voyages en Bulgarie], Sofia 1976, p.559.

16. Comparer avec la synthèse proposée dans Valchinova (Galia), «Znepolski pohvali ». Lokalna religija i identičnost $\mathrm{v}$ Zapadna Bălgarija [Religion locale et identité en Bulgarie occidentale], Sofia, 1999, p.46.

17. Voir Todorova (Maria), 1993, p.156, p.174.

18. Voir Petrov (G.), «Emigranskoto dviženie za Amerika v Makedonija » [Le mouvement d'émigration vers l'Amérique en Macédoine], Kulturno edinstvo, Sofia, 1909, p.3; Cvijić (Jovan), Balkansko poluostrvo i južnoslovenske zemlje [La péninsule balkanique et les pays slaves du sud], Knj. druga, Beograd, 1931, p.134, p.162, p.169, p.199.

19. Malheureusement pour les chercheurs d'aujourd'hui, les statistiques officielles bulgares, par exemple, ne comptabilisent pas, à quelque moment que ce soit de leur histoire, les migrations saisonnières de travail d'une durée inférieure à six mois(voir Economy of Bulgaria, 1969, p.408). Telle est la raison pour laquelle nous pouvons seulement fournir des estimations concrètes des migrations de travail temporaire vers ou depuis la Bulgarie.

20. Dans l'Empire ottoman jusqu'en 1912.

21. Petrović (J.), Pečalbari, naročito iz okoline Pirota, Beograd, 1920, p.23.

22. Hristov (Petko), « The Use of Holidays for Propaganda Purpose. The "Serbian" Slava and/or the "Bulgarian" Sabor ", Ethnologia Balkanica, 6, 2002, p.69-80.

23. Petrović (J.), Pečalbari, naročito iz okoline Pirota, Beograd, 1920, p.14.

24. Mironova-Panova (S.), Trănskijat kraj [La région de Trăn], Sofia, 1971, p.69-70.

25. Nikolić (V.), «Iz Lužnice i NišavE», Srpski etnografski zbornik, knj. 16, Beograd , 1910, p.28.

26. Petrichev (L.), "Trănskite dobrovolci v Srăbsko-turskata vojna - 1876 godina » [Les volontaires de Trăn dans la guerre serbo-turque en 1876], Trănski kraj, Sofia, 1940, p.140-162, p. 150.

27. Petrović (J.), Pečalbari, naročito iz okoline Pirota, Beograd, 1920, p.23.

28. Voir Petrichev (L.), « Trănskite dobrovolci v Srăbsko-turskata vojna - 1876 godina » [Les volontaires de Trăn dans la guerre serbo-turque en 1876], Trănski kraj, Sofia, 1940, p.140.

29. Op cit., p.163-171.

30. Petrović (J.), Pečalbari, naročito iz okoline Pirota, Beograd, 1920, p.23.

31. Op .cit., p.27.

32. Hristov (Petko), « Nikola Pasič i njegovo delovanje u emigraciji u Bugarskoj », Razvitak, $\mathrm{n}^{\circ}$. 213-214, Zaječar, 93, 2003, p.113-120.

33. Cvijić (Jovan), Osnove za geografiju i geologiju Makedonije i Stare Srbije, Beograd, 1906, p.197.

34. Petrov (G.), MAteriali za izučavaneto na Makedonija [Matériaux pour l'étude de la Macédoine], Sofia, 1896, p.593.

35. Koneska (E.), « Makedonci vo Istanbul » [Macédoniens à Istanbul], Etnolog, 1, 1992, Skopje, p. 64-72.

36. Cvijić (Jovan), Balkansko poluostrvo i južnoslovenske zemlje, кnj. druga, [La péninsule balkanique et les pays slaves du sud, livre 2], Beograd, 1931, p.135-136.

37. Voir Petrov (G.), «Emigranskoto dviženie za Amerika v Makedonija » [Le mouvement d'émigration vers l'Amérique en Macédoine], Kulturno edinstvo, Sofia, 1909, p.3-6.

38. A titre d'exemple, sur les 74 ouvriers dans la construction originaires du village de Radibuš (région de Kriva Palanka) qui travaillent à Sofia, 72 s'inscrivent en 1912 dans le mouvement de volontaires de Macédoine et de Thrace auprès de l'armée bulgare. 
39. Voir Kjushkosky (A.), Pečalbarstvoto vo Vevčani [Les pratiques pečalbar à Vevčani], Bitola, 1998, p.21.

40. Cvijić (J.), Naselja srpskih zemalja, Beograd, Državna štamparija, 1912, p.219.

41. Nikolić (R.), "Krajište i Vlasina », Srpski etnografski zbornik, knj. 18, Beograd, 1912, p.231; S. Mironova-Panova, Trănskijat kraj [La région de Trăn], Sofia, 1971, p.65. L'expression, venue du turc, est l'appellation dialectale de la grue.

42. Voir la collection de chansons folkloriques de Karovski (L.), Makedonski pečalbarski narodni pesni [Chansons populaires pečalbar macédoniennes], Skopje, 1979.

43. Pesheva (R.), «Edin starinen semeen praznik. Praznuvane na svetec v Severozapadnata i Zapadna Bălgarija » [Une veille fête familiale. La célébration d'un saint dans la Bulgazrie du NordOuest et de l'Ouest], Ezikovedski-etnografski izsledvanija $v$ pamet na akad. Romanski [Etudes ethnographiques et linguistiques en souvenir de l'Académicien Romanski], Sofia, 1960, p.739; Hristov (P.), - «Ahnenkult in Westbulgarien: das Fest des Schutzheiligen », in Brunnbauer (U.)., Kaser (K.), éds, Vom Nutzen der Verwandten. Soziale Netzwerke in Bulgarien (19. und 20. Jahrhundert), Böhlau, Wien, 2001, p.187-199.

44. Hristov (P.), - «Ahnenkult in Westbulgarien : das Fest des Schutzheiligen », in Brunnbauer (U.)., Kaser (K.), éds, Vom Nutzen der Verwandten. Soziale Netzwerke in Bulgarien (19. und 20. Jahrhundert), Böhlau, Wien, 2001, p.193.

45. Hobsbawm (E.), «Introduction: Inventing Traditions », in Hobsbawm (E.), Ranger (T.), eds, The Invention of Tradition, Cambridge University Press, 1983, p.1-14.

46. Au cours de mes recherches de terrain au cours de l'été 2005 , à Vevčani, j'ai visité la chapelle pour le «Spasovden pečalbar » avec des Gastarbeiter d'Allemagne ayant pris leur retraite et entrepris de la restaurer.

47. Voir, sur le cas de Mala Reka, Resova (M.), « Običai na Mijacite-gurbetčij ot Mala Reka » [Les coutumes des personnes pratiquant le gurbet originaires de la région de Mijak, de Mala Reka], Vesnik na Muzejsko-konzervatorskoto društvo na NRM, 1, Skopje, 1955, p.7-14; Spirovska (L.), «Za nekoj mijački pečalbarski običai pocebno ot Galičnik» [De quelques coutumes pečalbar de la région de Mijak, en particulier de Galičnik], Bitorski naučno-kulturni sobiri I, Gostivar, 1971, p. 259-263.

48. Irechek (K.), Istorija na Bălgarite [Histoire des Bulgares], Sofia, 1978, p.48.

49. Au cours des dernières décennies du XXème siècle, le mariage à Galičnik s'est transformé en une représentation folklorique. Jusqu'à aujourd'hui, cependant, les personnes qui souhaitent réellement se marier à Galičnik ne peuvent le faire dans l'église locale que le jour de la Saint Petrov. Au cours de l'été 2005, j'ai assisté à trois mariages consécutifs à Galičnik lors de la Saint Petrov.

50. Les relations entre les nouveaux Etats nés de l'effondrement de la Yougoslavie et la diaspora en Europe de l'ouest des Gastarbeiter constituent un thème intéressant qui mériterait de faire l'objet d'une étude séparée.

51. Hristov (P.), « Granicite na "Šopluka" i/ili šopi bez granici » [Les frontières du « Šopluk » et/ ou les Šops sans frontières], in Skrivene manjine na Balkanu [Les minorités caches dans les Balkans], Beograd, 2004, p.117-118.

52. Les premières études historiques, culturelles et ethnologiques relatives à ces migrations dans la région des Rhodopes en Bulgarie sont d'ores et déjà disponibles. Voir l'ouvrage collectif dirigée par Margarita Karamihova joliment intitulé, Da živeeš tam, da se sănovaš tuk [Vivre là-bas, se rêver ici], Sofia, 2003. 


\section{RÉSUMÉS}

L'article analyse, sur la base de sources historiques et de matériaux de recherche collectés à l'occasion de terrains effectués en Bulgarie occidentale et en Macédoine, l'histoire, les destinations (aussi bien à l'intérieur de la péninsule balkanique, y compris en Valachie/au sud de la Roumanie, qu'en direction de l'Europe et de l'Amérique) et les traditions de ce qu'il est convenu d'appeler le gurbet, à savoir les mobilités de travail temporaires, principalement masculines, de la seconde moitié du XIXème siècle et de la première moitié du XXème siècle. L'accent est placé sur la manière dont ces expériences de travail saisonnier dans le cadre du gurbet ont influé sur les modèles culturels traditionnels en matière d'organisation sociale, de rituels du cycle de vie ou de fêtes calendaires. Cette perspective historique apparaît indispensable à l'intelligibilité des représentations et des pratiques de la mobilité de travail observées dans la seconde moitié du XXème siècle, singulièrement à partir des années 1960 et 1970 - non pour postuler l'existence d'une continuité entre ces deux périodes historiques, mais pour mieux saisir les conditions de transformations des répertoires de la mobilité.

\section{INDEX}

Index géographique : Bulgarie, Macédoine, Balkans, Serbie

Mots-clés : migrations, Gurbet, migrations saisonnières

\section{AUTEURS}

\section{PETKO HRISTOV}

Dr. Petko Hristov est Directeur de recherche à l'Institut et au Musée d'ethnographie de l'Académie des sciences bulgares (BAN). Diplômé en ethnographie de l'Université de Sofia « Kliment Ohridski », il a soutenu en 1997 une thèse sur les « Normes de comportement dans le village traditionnel bulgare dans la Bulgarie du Centre-Ouest au début du XXème siècle ». Son habilitation, défendue en 2005, portait sur «Communautés et fêtes». Professeur associé à l'Université du Sud-Ouest « Neofit Rilski » de Blagoevgrad depuis 2006, ses recherches concernent les institutions et normes populaires de comportement, les familles et relations de lignage, les définitions sociales des rôles dans la société bulgare traditionnelle, ainsi que les migrations de travail, singulièrement dans une perspective historique. Il a récemment publié : " Trans-border Exchange of Seasonal Workers in the Central Part of the Balkans », Ethnologia Balkanica, 12, 2008 (à paraître); «Celebrating the Abandoned Village (pusto selo): Pictures from the Ritual Process in the Post-Socialist Balkans ", in Biljana Sikimić and Petko Hristov (eds), The Kurban on the Balkans, Belgrade, Institute for Balkan Studies, 2007, p.245-258; « Places to Exchange Cultural Patterns : The Market and the Piazza for Hired Labour in Sofia », Ethnologia Balkanica, 9, 2005, p.81-90.

mhristov_p@yahoo.com 